Meta

Journal des traducteurs

Translators' Journal

\title{
La nature et l'optique expressive
}

\section{Yvonne St-Pierre Farina}

Volume 20, numéro 3, septembre 1975

URI : https://id.erudit.org/iderudit/002730ar

DOI : https://doi.org/10.7202/002730ar

Aller au sommaire du numéro

Éditeur(s)

Les Presses de l'Université de Montréal

ISSN

0026-0452 (imprimé)

1492-1421 (numérique)

Découvrir la revue

Citer cet article

St-Pierre Farina, Y. (1975). La nature et l'optique expressive. Meta, 20(3),

194-205. https://doi.org/10.7202/002730ar

Ce document est protégé par la loi sur le droit d'auteur. L'utilisation des services d'Érudit (y compris la reproduction) est assujettie à sa politique d'utilisation que vous pouvez consulter en ligne.

https://apropos.erudit.org/fr/usagers/politique-dutilisation/
Cet article est diffusé et préservé par Érudit.

Érudit est un consortium interuniversitaire sans but lucratif composé de l'Université de Montréal, l'Université Laval et l'Université du Québec à Montréal. Il a pour mission la promotion et la valorisation de la recherche. https://www.erudit.org/fr/ 


\section{La nature et l'optique expressive}

\section{LA CLASSIFICATION SELON LA NATURE}

Vinay et Darbelnet, dans leur Stylistique comparée du français et de l'anglais ', ont conclu que la langue fonctionne sur DEUx niveaux : le plan du réel et le plan de l'entendement. Pourtant, les éléments expressifs tels que présentés dans un article précédent, font songer à la possibilité d'un fonctionnement sur plusieurs niveaux. La difficulté provient de ce que le même terme peut cumuler plusieurs couches expressives. Comment peut-on distribuer par catégories, en allant du moins au plus, dans de telles conditions? Quels seraient les critères utiles à cette fin?

Bally a suggéré ${ }^{2}$, sans avoir pu en faire l'application, deux critères nécessaires. Sous certains rapports il s'est même contredit. N'empêche qu'il semble avoir vu juste. Ces critères se résument à deux : la dominance et la primauté du concret.

a) La dominance. - Étant donné que plusieurs éléments expressifs peuvent se trouver dans le même terme, il faut déterminer l'ordre des catégories expressives par la dominance, à savoir l'élément expressif prépondérant.

b) La primauté du concret. - Ce critère vient modifier le premier. Puisque le mot peut contenir deux dominances, il faut que la dominance affective cède à la dominance concrète. C'est le cas de hissed, ooze out, hurl, en anglais ; et du français hurler. Ces termes vont se grouper par leur contenu concret non par leur affectivité car celle-ci est trop floue et trop universelle pour fournir un fondement suffisamment stable pour la classification. L'affectivité peut imprégner, par le geste, l'intonation, la ponctuation, la situation, la syntaxe expressive, le mot le plus intellectualisé alors que le concret détaillé ne pénètre que là où le permet l'esprit collectif. Ce second critère vient donc agir en contrepoids au premier.

\section{Lavue}

Avant de pouvoir différencier et classer, il faut découvrir le dénominateur commun. Celui-ci est constitué par la vue. L'homme voit la réalité et la nomme. Cette vue, en linguistique, on l'applique, par métonymie, au mot lui-même.

1. Jean-Paul Vinay et Jean Darbelnet, Stylistique comparée du français et de l'anglais, Paris, Didier, 1958 , p. 58-74. 2. Charles Bally, Traité de stylistique française, $3^{\circ}$ ed., Genève et Paris, Georg et Klincksieck,
1951, vol. I, p. 140-250. 
a) La vue représentative. - La vision tend vers l'abstraction, le général, lo neutre ou l'impartial. Le mot est à dominance conceptuelle. C'est le niveau le plus bas par rapport au concret : la terre est ronde; le soleil brille; j'existe; je vais' au bureau à pied. Chaque vue dans ces phrases est incolore et générale. Elle est prosaïque et conventionnelle, sans accentuation de détail. Son rôle principal est de représenter le concept.

b) La vue émotive. - Ces vues montent d'un degré dans la concrétivité. Sans insister sur le détail pittoresque, elles emportent notre adhésion ou nous détournent de telle réalité. Des mots tels que sloppy, delightful, elated, tantalize, wee, loathe, detestable, diabolical, traitorous, forlorn, et, en français, hideux, hilarite, femmelette, fripon, polisson, affliction, doucereux, une popote, une guimbarde, contiennent déjà un élément concret plus chaud, plus expressif, sans toutefois fournir des détails précis. $\mathrm{Si}$ je dis que cet enfant est un laideron, rien ne m'indique précisément la source de cette laideur : est-ce le nez, les oreilles, les yeux? Laideron est un terme à dominance affective où l'élément concret est secondaire mais plus précis que dans les vues représentatives.

La vue émotive révèle des attitudes. «Tu es béni du Seigneur» ou « le Seigneur te maudira». Cette vue peut exprimer l'intensité : «tes oreilles sont énormes» ou «tes oreilles sont minuscules». L'on voit par ces exemples que les attitudes peuvent être positives ou négatives et que l'intensité peut être augmentative ou diminutive. Nous pouvons conclure donc que l'émotivité est bivalente, c'est-à-dire qu'elle contient en elle deux orientations, deux valeurs, l'une étant le contraire de l'autre. L'expérience commune nous renseigne d'ailleurs sur la bivalence des attitudes et de l'intensité.

c) La vue concrète. - Celle-ci constitue le dernier échelon dans la hiérarchie de la concrétisation. Ce sont les mots-images (les mots-qui-font-image). Ces vues précisent le détail, souvent d'une façon pittoresque. La vision est précise. Des vues telles que rap, snap, tap, click, clash, rayonner, miroiter, papilloter, chatoyer, apportent une vue du réel plus particularisée. Ces vues séduisent surtout les sens et l'imagination : below, spicy, splutter, aromatic, silvery, leap; lancer, bondir, arôme, frôler, feuilleter.

La vue concrète est parfois plus affective que certaines vues émotives. "The blood oozed out $»$. Ici l'expression révèle un jugement de valeur très péjoratif. L'affectivité constitue donc l'une des dominantes. Mais puisque le concret fournit notre critère de hiérarchisation, et puisque les modalités particularisantes présentent un concret extrêmement saillant (la lenteur de l'écoulement; une moiteur de la surface; passage du sang à travers les pores; l'idée d'abondance), on range ooze out parmi les vues concrètes plutôt que parmi les vues émotives.

\section{Les sigles expressifs}

Par la suite nous allons utiliser les lettres $A, B, C$, placés directement après le mot qui intéresse notre propos, pour indiquer les différentes vues. Ainsi :

$$
\begin{aligned}
& \mathbf{A}=\text { Les vues représentatives. } \\
& \mathbf{B}=\text { Les vues émotives. } \\
& \mathbf{C}=\text { Les vues concrètes. }
\end{aligned}
$$


Par exemple, bondir (C) voudra indiquer que bondir est une vue concrète; sauter (A) que sauter est une vue représentative, etc. (Pour faciliter la rétention mémorielle de ces sigles l'on pourrait faire les associations suivantes : $\mathrm{C}=$ concret $; \mathrm{A}=$ Abstrait $; \quad B=$ sivalent ).

Voilà donc la hiérarchie fondamentale qui se dissimule à l'intérieur du réseau d'associations mémorielles. Cette hiérarchie s'applique aux deux langues, le français et l'anglais, mais chacune aura ses préférences et ses particularités.

\section{Passage d'une langue à l'autre}

La nature de la vue correspond très souvent à celle de son homonyme dans l'autre langue, à témoin les exemples suivants :

Un excellent (B) portrait (A), soit.

An excellent (B) portrait (A), granted ${ }^{3}$.

- C'est qu'il m'arrive une histoire abominable (B) ...

«Something abominable (B) had happened to $\mathrm{me}^{4}$."

"She is of an unbelievable (B) barbarousness (B).»

- Elle est d'une violence (B) incroyable (B) ${ }^{5}$.

Cunning (B) and serene (B).

Rusé (B) et serein (B) ${ }^{6}$.

We climbed the fence, where the pigs grunting (C) and snuffing (C).

Nous avons grimpé la barrière là où les cochons grognaient $(\mathrm{C})$ et reniflaient (C) ${ }^{7}$.

We went through the rattling $(\mathrm{C})$ leaves.

Nour marchions dans les feuilles bruissantes (C) 8 .

... Tu me tapotais (C) la joue...

... You patted (C) my cheeks... ${ }^{9}$

À dix heures elle s'éveilla, toute frileuse (C) et engourdie (C).

At ten o'clock she awoke, shivering (C) and benumbed (C) ${ }^{\circ}$.

3. Jean-Paul Sartre, la Nausée, Paris, Le Livre de Poche, 1968, p. 133 ; Nausea (traduction par Lloyd Alexander), New York, New Directions Publishing Co., 1964, p. 126.

4. Jean-Paul Sartre, la Nausée, p. 110 et 104.

5. Enest Hemingway, For Whom the Bell Tolls, New York, Scribners, 1940, p. 28 ; Pour qui sonne le glas (traduction par Denise von Mappès), Paris, Le Livre de Poche, 1968, p. 34.

6. William Faulkner, The Sound and the Fury, New York, Random House, 1948, p. 95; le Bruit et la fureur (traduction par Maurice E. Coindreau, Paris, Le Livre de Poche, 1949, p. 117.

7. Ibid., p. 3, 23.

8. Ibid.,p. 4, 24.

9. François Mauriac, le Noud de vipères, Paris, Le Livre de Poche, 1933, p. 13 ; Vipers' Trangle (traduction par W. B. Wells), Garden City, New York, Image Books, 1957, p. 14.

10. Germaine Guèvremont, le Survenant, Montréal et Paris, Fides, 1962, p. 160 ; The Outlander (traduction par Eric Sutton), New York, Whittlesay House, 1950, p. 82. 
À peu de choses près, la nature expressive se correspond dans les deux langues en présence. Nous sommes en face, ici, de passages $\mathrm{A} / \mathrm{A}, \mathrm{B} / \mathrm{B}, \mathrm{C} / \mathrm{C}$. Cette nature expressive, cependant, ne se place pas toujours sur le même échelon expressif, comme nous le verrons dans les exemples suivants :

L'eau se retira (A) des rigoles.

The water trickled out $(\mathrm{C})$ of the gutters ${ }^{11}$.

... on entendait des bruits (A) d'assiettes et de couverts.

One could hear a faint timkle (C) of knives and forks and crockery... ${ }^{12}$

Le grand corps de Denis apparut (A) derrière.

Denis' big body loomed $(\mathrm{C})$ behind her ${ }^{13}$.

[le vent] ... gonflait $(\mathrm{C})$ les rideaux, refraîchissant mes yeux ...

[the wind] ... filled (A) the curtains and bathed my eyes ${ }^{14}$.

... déjà la terre les englue $(C)$ par en-dessous.

... the earth has already caught (A) them below ${ }^{15}$

(en parlant des feuilles mortes).

Quelquefois je les palpe (C) simplement en les regardant de tout près... Sometimes I feel them (A) looking at them closely... ${ }^{16}$

Nous étions en pleine (B) veillée.

We were all smug (C) for the evening ${ }^{17}$.

... et les grands silences inquiets (B) de ses nuits l'aiguillonnaient au travail. $\ldots$ and the throbbing (C) silence of the night spurred him on to work ${ }^{18}$.

À intervalles fréquents, une cloche éraillée (C) sonnait en haut de la rue Notre-Dame et un tram passait (A).

At frequent intervals a bell clanged harshly (B) up Notre Dame street and a trolley swayed by (C) ${ }^{19}$.

Je devais faire de la vie la palpitante $(\mathrm{C})$ découverte.

The thrilling (B) discovery of life was to be mine ${ }^{20}$.

[Denis veut tuer la poule] Mais la pauvre bête s'enfuyait étonnée de ce que ce grand indifférent s'occupât (A) d'elle avec tant d'insistance, tout à coup.

11. Germaine Guèvremont, le Survenant, p. 177 et 91.

12. Albert Camus, l'Etranger, Paris, Le Livre de Poche, 1968, p. 80 ; The Stranger (traduction par Stuart Gilbert), New York, 1946, p. 67.

13. Roger Lemelin, Au pied de la pente douce, Montréal, Editions de l'Arbre, 1944, p. 133 The Town Below (traduction par Samuel Putnam), Toronto, McClellan and Stewart, 1961, p. 116.

14. François Mauriac, le Neud de vipère, p. 52 et 44.

15. Jean-Paul Sartre, la Nausée, p. 21 et 19.

16. Ibid.

17. Alain-Fournier, le Grand Meaulnes, Paris, Editions Emile-Paul, 1966, p. 120 ; The Wanderer (traduction par Françoise Delisle), New York, Doubleday, 1953, p. 102.

18. Gabrielle Roy, Bonheur d'occasion, Paris, Flammarion, 1945, p. 37 ; The Tin Flute (traduction par Hannah Josephson), Toronto, McClellan and Stewart, 1961, p. 16.

19. Ibid., p. 91 et 49.

20. André Gide, l'Irmmoraliste, Paris, Le Livre de Poche, 1965, p. 31; The Immoralist (traduction par Dorothy Bussy), New York, Random House, 1930, p. 19. 
But the poor creature, astonished that this big fellow who had never paid any attention to her before, should suddenly woo (B) her so insistently, darted out of reach ${ }^{21}$.

La dernière série d'exemples nous fournit les passages suivants :

se retira/trickled out $=\mathrm{A} / \mathrm{C}$

bruits $/$ tinkle $=\mathrm{A} / \mathrm{C}$

apparut/loomed $=\mathrm{A} / \mathrm{C}$

gonflait $/$ filled $=\mathrm{C} / \mathrm{A}$

englue $/$ caught $=\mathrm{C} / \mathrm{A}$

palpe $/$ feel $=\mathrm{C} / \mathrm{A}$

en pleine $/$ smug $=\mathrm{B} / \mathrm{C}$

inquiets $/$ throbbing $=\mathrm{B} / \mathrm{C}$

éraillée/harshly $=\mathrm{C} / \mathrm{B}$

passait/swayedby $=\mathrm{A} / \mathrm{C}$

palpitante/thrilling $=\mathrm{C} / \mathrm{B}$

s'occupât $/$ woo $=\mathrm{A} / \mathrm{B}$

De telles différences de nature expressive abondent en passant d'une langue à l'autre. Leur importance provient du fait que chacune des deux langues a ses préférences, démarches dont le traducteur doit tenir compte sous peine d'altérer la tonalité du texte original.

Il importe, en traduisant, de comprendre les relations existant entre les vues. La vue représentative n'est, évidemment, pas une image. Elle est trop abstraite, trop conceptuelle. Elle se situe sur le plan de l'entendement. Son affectivité, toujours accidentelle et accessoire, lui est fournie surtout par le contexte ou la situation, par l'intonation, le ton, le geste, etc. Ce sont ces caractéristiques qui l'opposent radicalement à l'image. Son utilité provient du fait qu'elle agit en contrepoids à la vue émotive et la vie concrète.

La vue émotive ne constitue pas, elle non plus, une image mais elle est en puissance d'image. L'embryon d'image qu'elle contient n'émerge jamais complètement dans le domaine du précis.

Les vues concrètes, au contraire, forment toujours une image. Elles précisent, elles détaillent, elles s'adressent aux sens. Elles sont souvent pittoresques.

Il appert donc que la langue fonctionne sur trors plans et non sur deux, comme l'affirment Vinay et Darbelnet. Ce serait le plan de l'entendement, le plan de l'émotivité et le plan du réel. La notion d'optique viendra toutefois modifier cette conclusion.

\section{LA CLASSIFICATION SELON L'OPTIQUE}

Le point de repère le plus important pour l'évaluation de la nature expressive des vues nous est fourni par le dosage de relief concret. Ce critère peut d'abord paraître arbitraire, mais si l'on songe aux relations très étroites existant entre les

21. Roger Lemelin, Au pied de la pente douce, p. 153 et 135. 
enregistrements des sens, l'usage de l'intelligence et les manifestations de la sensibilité, l'on constatera forcément la justesse de ce choix. Cependant le relief concret n'aura pas le dernier mot : il faut nécessairement faire intervenir un autre contrôle : l'angle de vision, l'optique sous laquelle on perçoit l'expressivité de cette réalité, qu'elle soit représentative, émotive ou concrète.

Voilà donc le troisième critère de catégorisation, à savoir l'optique : la vision d'une réalité par rapport à sa présence ou à son absence de sa situation coutumière, et le circuit sémantique que peut obliger la confrontation de l'esprit avec telle réalité présente dans une situation habituelle. Nous sommes en face de ce que l'on appelle communément a) le sens propre, b) la comparaison, c) la métaphore.

Cet angle de vision n'a rien de commun avec l'angle de vision de tel poète, de tel auteur. Cet angle de vision est un fait de langue qui étudie le mot replacé dans son contexte, la phrase, l'énoncé. Son étude a son importance car les langues ont chacune leur angle de vision préféré. Souvent une traduction, par ailleurs parfaitement correcte, «sent » la traduction parce qu'elle suit constamment l'optique de la langue de départ. Elle est remplie d'écarts expressifs tellement subtils que seul le spécialiste saura les discerner.

\section{Les trois optiques}

a) Il existe trois optiques possibles, dont la première est la vue directe : la réalité nous apparaît dans sa situation habituelle, sans l'intervention d'une autre réalité avec laquelle on la compare pour en compléter la signification ou l'expressivité :

\section{La tasse est sur la soucoupe.}

b) La seconde optique est constituée par la comparaison. C'est la vue oblique:

Elle est bonne COMME du pain blanc.

Il est rusé COMME un renard.

La première réalité (la bonne personne, l'individu rusé) est présente dans sa situation coutumière. Par l'entremise du pivot de comparaison COMME, on introduit de façon explicite une seconde réalité qu'on arrache de sa situation habituelle pour la replacer dans celle d'où elle est normalement absente (le plain blanc, le renard). Il est résulte une autre perception de la réalité : une perception non pas directe mais oblique, c'est-à-dire vue par rapport à une autre réalité. On voit, par exemple, par rapport au pain blanc, la bonté.

c) La métaphore constitue la troisième optique. L'optique métaphorique va plus loin que l'optique oblique. Le regard finit par fusionner les deux réalités dont l'une seulement est présente dans sa situation. Le regard porte directement sur la réalité normalement absente de la situation et indirectement sur celle qui y est habituellement :

Mathilde $[\ldots]$ guettait l'orage montant des deux voix confondues... ${ }^{22}$

22. François Mauriac, Génitrix, Paris, Lo Livre de Poche, 1964, p. 35. 
Ici l'on déplace l'orage pour le transporter dans un contexte inhabituel. La colère est vue indirectement par l'entremise de la violence d'un phénomène naturel. Ce déplacement de la réalité dote la métaphore de son dynamisme, mais le contexte étant en somme une option, la couche affective qui en résulte est accidentelle.

\section{Les sigles}

Nous allons utiliser les chiffres $1,2,3$, pour représenter l'optique :

1. L'optique directe (le sens propre).

2. L'optique oblique (la comparaison).

3. L'optique métaphorique (la métaphore).

Placés à côté des lettres $\mathrm{A}, \mathrm{B}, \mathrm{C}$, qui nous indiquent la nature expressive, les chiffres $1,2,3$, vont dispenser des long commentaires qui autrement entraveraient ou même empêcheraient une étude détaillée des plans sur lesquels la langue fonctionne. Ainsi :
A $1=$ vue représentative directe.
A 2 = vue représentative oblique.
A $3=$ vue représentative métaphorique.
B $1=$ vue émotive directe.
B 2 = vue émotive oblique.
B $3=$ vue émotive métaphorique.
$\mathrm{C} 1=$ vue concrète directe.
$\mathrm{C} 2$ - vue concrète oblique.
C 3 = vue concrète métaphorique.

L'utilité de ces sigles deviendra évidente dans les exemples que nous présentons à l'appui de notre théorie.

\section{Passage d'une langue à l'autre.}

Toutes sortes de possibilités se présentent. Dans le premier cas on modifie la nature, mais pas l'optique :

... la cour sèche, sans (A 1 ) paille...

... a dissued yard destitute of (B 1) straw... ${ }^{23}$

[II] ne se dégoûtait pas (B 1) de l'étude.

[He] was not tired (A 1) of study ${ }^{24}$.

Les dents d'acier entamèrent (A 1) la plane.

$\ldots$ and the steel teeth hissed (C 1 ) into the plane-tree $\operatorname{logs}{ }^{25}$.

Ses tempes battaient (A 2), dans une montée (A 2) de sang, AINSI QUE sous les coups de deux mains acharnées

... her temples were throbbing (C 2) in a surge (C 2) of blood AS THOUGH two harsh hands were beating them ${ }^{26}$.

23. Alain-Fournier, le Grand Meaulnes, p. 128 et 107.

24. Roger Lemelin, Au pied de la pente douce, p. 186 et 162.

25. Germaine Guèvremont, le Survenant, p. 37 et 17 .

26. Ibid., p. 38 et 17 . 
Et parfois le vent IMITAIT dans les frondaisons (C 2) le bruit d'une averse (C 2).

Sometimes the wind in the leaves (A 2) IMITATED the sound of rain (A 2) ${ }^{27}$.

Didace se sentit fier et un reste (A 3 ) de joie colla à lui.

Didace felt proud, and joy flickered up (C 3) again within him ${ }^{28}$.

... la mer haletait (C 3) de toute la respiration (A 3) rapide (A 3) et étouffée (3) de ses petites vagues.

... and small waves were lapping (C 3 ) the hot sand in little (B 3 ) flurried (C 3 ) gasps (C 3$)^{20}$.

Une énumération des passages présentés nous fait déjà soupçonner la très grande variété de passages possibles. Souvenons-nous que dans ceux-ci l'optique ne se modifiait pas :

sans $/$ destitute of $=A 1 / B 1$.

se dégoûtait/was tired $=\mathrm{B} 1 / \mathrm{A} 1$.

entamèrent $/$ hissed $=\mathrm{A} 1 / \mathrm{C} 1$.

battaient/were throbbing $=\mathrm{A} 2 / \mathrm{C} 2$.

montée $/$ surge $=\mathrm{A} 2 / \mathrm{C} 2$.

frondaisons/leaves $=\mathrm{C} 2 / \mathrm{A} 2$.

averse $/$ rain $=\mathrm{C} 2 / \mathrm{A} 2$.

reste/flickered up $=\mathrm{A} 3 / \mathrm{C} 3$.

haletait/lapping $=\mathrm{C} 3 / \mathrm{C} 3$.

respirations $/$ gasps $=\mathrm{A} 3 / \mathrm{C} 3$.

rapide/flurried $=\mathrm{A} 3 / \mathrm{C} 3$.

rapide $/$ little $=$ A $3 / \mathrm{B} 3$.

Dans les cas suivants la nature expressive du mot restera la même en passant d'une langue à l'autre. Seule l'optique se modifiera :

Quand Raymond m'a donné son revolver, le soleil a glissé (C 3) dessus.

The sun glinted (C 1) on Raymond's revolver as he handed it to $\mathrm{me}^{30}$.

La petite sonnette $[\ldots .$.$] grelottait (C 3$ ) au moindre souffle du vent.

The little bell [...] tinkled (C 1) over every breath of wind ${ }^{31}$.

[le ton] [...] se révéla bourré (C 3) d'anxiété...

[her voice] [...] actually quavered (C 1 ) ... ${ }^{32}$

... les marches geignaient (C 3 ) sous son pas pesant...

... the steps creaked (C 1 ) beneath his ponderous tread ${ }^{33}$.

Les étoiles de l'aube palpitaient (C 3 ) encore.

The stars were twinkling (C 1 ) before the dawn ${ }^{34}$.

27. François Mauriac, le Naud de vipères, p. 50 et 43 .

28. Germaine Guèvremont, le Survenant, p. 138 et 70.

29. Albert Camus, l'Etranger, p. 87 et 73 .

30. Ibid., p. $85-86$ et 72 .

31. Gabrielle Roy, Bonheur d'occasion, p. 63 et 32 .

32. Germaine Guèvremont, le Survenant, p. 218 et 111.

33. Ibid., p. 254 et 130 .

34. François Mauriac, le Neud de vipères, p. 52 et 44. 
Tous ces exemples constituent des passages $\mathrm{C} 3 / \mathrm{C} 1$, mais il y a aussi des passages :

A3/A1 : ... Colin connaissait son maigre (A 3) salaire. ... Colin knew how small (A 1 ) his salary was ${ }^{35}$.

B3/B1 : Il déchira une peau tenace (B 3) à petits coups de dents. He nibbled away at a particularly tough (B 1) cuticle ${ }^{36}$.

$\mathrm{C} 2 / \mathrm{C} 3$ : Il eut des gestes larges, comme pour tout bousculer (C 2). ... he felt that he could sweep (C 3 ) everything before him if he chose $^{37}$.

B1/B2 : ... un chant bizarre (B1) me fit tout à coup retourner. ... a noise... which sounded LIKE the bass accompaniment to a curious (B2) kind of singing, made me look around abruptly ${ }^{38}$.

Les cas les plus intéressants, évidemment, vont se présenter lorsque la nature et l'optique changent toutes les deux. Ainsi :

C3/B1 : Sentir ma première leçon mal comprise avait éperonné (C3) mon désir...

The feeling that my first lesson had been misunderstood stimulated (B1) me... ${ }^{39}$.

B3/C1 : Le ciel s'attristait (B3).

The sky was overcast $(\mathrm{C} 1)^{40}$.

Venant, la chevelure déjà en révolte (B3).

... his hair already ruffled $(\mathrm{C} 1)^{41}$.

A3/B1 : ... capable de chaude (A3) amitié pour la terre.

... devoted (B1) to the soil ${ }^{42}$.

B3/A1 : [les oiseaux] volaient en herse par bandes de cinquantaine... d'un vol tourmenté (B3).

They flew hrarow-wise in companies of fifty... in rather agitated (A1) flight ${ }^{43}$.

$\mathrm{A} 3 / \mathrm{C} 1$ : ... la galette de sarrasin grise et pivelée, aux cent yeux (A3) vite ouverts (A3) par la chaleur.

... the gray and lumpy buckwheat hough, soon to sputter (C1) into a thousand blisters (C3) over the fire ${ }^{44}$.

35. Roger Lemelin, Au pied de la pente douce, p. 191 et 165-166.

36. Gabrielle Roy, Bonheur d'occasion, p. 66 et 34 .

37. Roger Lemelin, Au pied de la pente douce, p. 70 et 65.

38. André Gide, l'Immoraliste, p. 71 et 51 .

39. Ibid., p. 111 et 86 .

40. Germaine Guèvremont, le Survenant, p. 150 et 76 .

41. Ibid., p. 206 et 105.

42. Ibid., p. 62 et 30 .

43. Ibid., p. 76 et 37 .

44. Ibid., p. 50 et 23 . 
A1/C3 : Et de l'avoir ainsi jugé dans son esprit lui procura une minute (A1) de véritable satisfaction.

And it gave her a spark (C3) of satisfaction to have judged him so severely in her mind ${ }^{45}$.

Nature et optique entrent dans un jeu perpétuel comme si elles s'adonnaient ainsi à une politique d'accommodement où les concessions mutuelles limitent ou étendent les préférences de chaque langue. Cependant une étude de ce jeu entre la nature et l'optique prolongerait démesurément cet article.

De ces exemples qui abondent dans la langue, nous pouvons tirer certaines conclusions. D'abord, la langue directe (le sens propre) se ramène à trois niveaux expressifs, l'un comportant plus de concret que l'autre :

a) Les vues représentatives directes.

b) Les vues émotives directes.

c) Les vues concrètes directes, ou les mots-images directs.

Chacune de ces vues, lorsqu'on l'incorpore dans une comparaison, revalorise les éléments expressifs qui étaient siens au niveau direct. Nous nous trouvons visà-vis trois autres niveaux expressifs :

a) Les vues représentatives obliques.

b) Les vues émotives obliques.

c) Les vues concrètes obliques ou les mots-images obliques.

La comparaison ajoute une, parfois deux, couches expressives à l'expressivité qui y existait déjà.

Quant à la métaphore, elle se présente sur trois paliers de concret différents. Les métaphores n'ont pas toutes, par conséquent, la même valeur expressive :

a) La vue représentative métaphorique (la plus conceptuelle de toutes).

b) La vue émotive métaphorique (à dominance affective, concret imprécis).

c) La vue concrète métaphorique (le mot-image).

Les trois natures s'échelonnent chacune d'elles sur trois niveaux expressifs, Par conséquent, la langue fonctionne sur NEUF niveaux expressifs, et non pas sur les deux niveaux seulement qu'expliquent Vinay et Darbelnet.

Voilà les neuf niveaux expressifs entre lesquels il est important de distinguer, puisque l'anglais et le français favoriseront, les uns aux dépens des autres, chaque échelon comportant certains éléments expressifs susceptibles d'attirer la faveur du sujet parlant, ou de lui répugner. Il va sans dire que ce choix s'opère inconsciemment d'après une intuition développée par l'usage quotidien de la langue. Si l'homme qui parle forge la langue, elle le forme à son tour.

45. Gabrielle Roy, Bonheur d'occasion, p. 36 et 10. 
Le tableau suivant représente cette hiérarchisation des vues expressives.

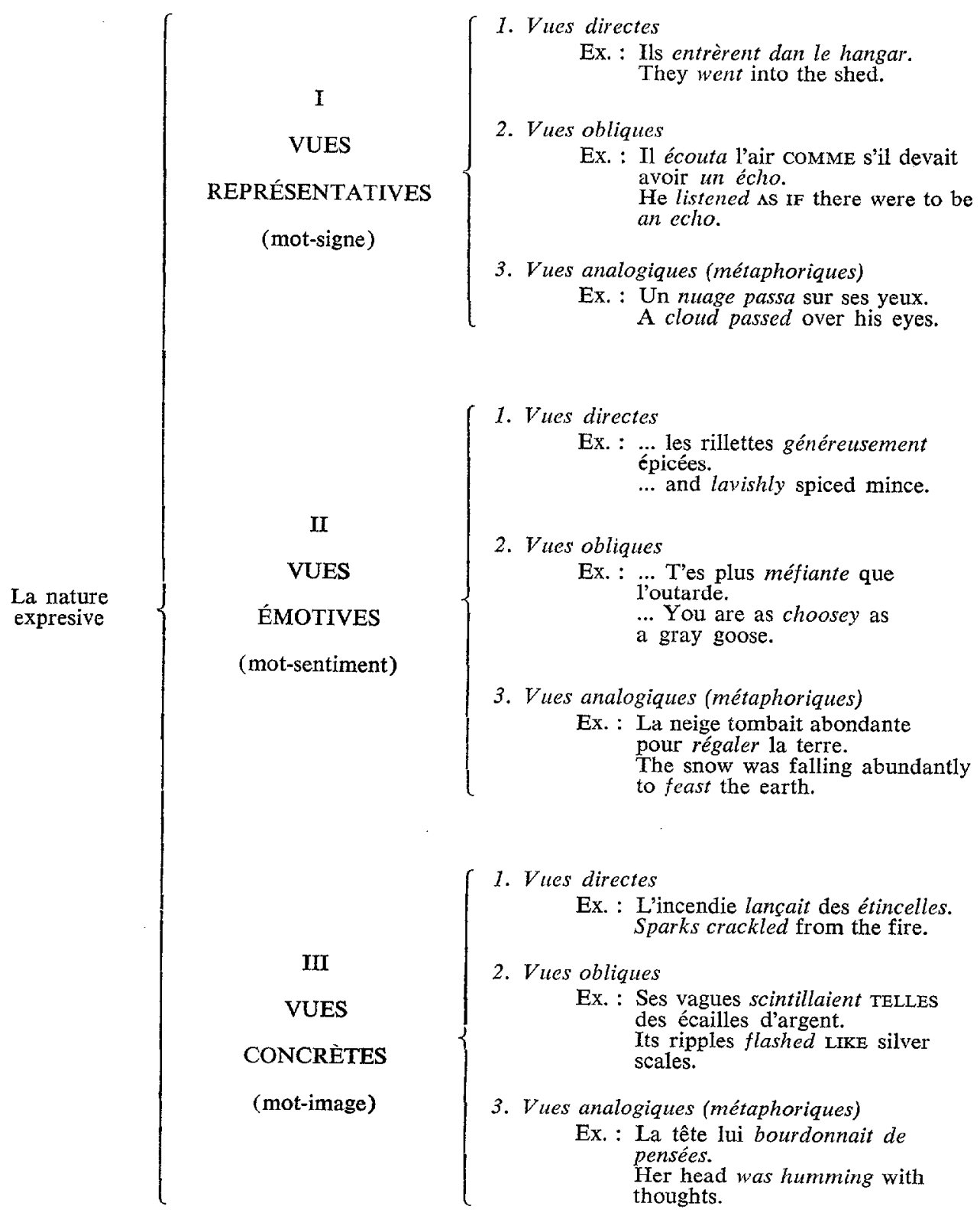

Cette conclusion ne contredit pas le résultat du travail des MM. Vinay et Darbelnet, mais elle y apporte de très importantes modifications. Le plan de l'entendement, tel que le conçoivent ces deux auteurs, utilise le mot signe, dans notre étude, la vue représentative. Le plan du réel, selon les mêmes auteurs, emploie le 
mot-image : pour nous la vue concrète. Le plan émotif est donc un autre plan où interviennent les mots dits d'ambiance - la vue émotive - et ce plan se situe entre le plan de l'entendement et le plan du réel.

Encore faut-il se rendre compte que dans leur ouvrage le plan de l'entendement et le plan du réel comportent souvent un sens large : lancer et hurl sont tous les deux sur le plan du réel, mais lancer par rapport à hurl se trouve sur le plan de l'entendement parce qu'il comporte un degré d'abstraction supérieur à hurl, lequel est plus près du réel que son correspondant français.

Ou encore : houses inhabited by drunkards, est sur le plan du réel comparativement à « des maisons $\grave{a}$ ivrogne », bien qu'absolument les deux termes soient sur le plan de l'entendement. À ce moment-là les limites entre les deux plans se déplacent et «entendement » prend plus d'extension que «mot-signe » ou «vue représentative », puisqu'il peut, par rapport à une seconde vue plus détaillée, se rapporter à un concret plus détaillé. Pour les mêmes raisons, «plan du réel » peut comprendre des vues représentatives. Ce n'est que lorsque "plan d'entendement 》 et «plan du réel » se prennent au sens strict qu'ils ont la même extension que «mot-signe» et «vue concrète » respectivement. Le plan émotif pourrait, lui aussi, prendre plus d'extension que la vue émotive, puisqu'un mot comme to prowl est en même temps un mot-image et un mot affectif.

Les trois plans, au sens strict, correspondent aux vues représentatives, aux vues émotives et aux vues concrètes; mais notons encore une fois que ces trois plans peuvent prendre trois orientations différentes : l'optique directe, l'optique oblique et l'optique analogique.

Enfin, les cadres expressifs ne font qu'organiser l'échelle expressive déjà dans le subconscient. Mais ils vont fournir une méthode d'analyse expressive non seulement au traducteur mais au stylisticien en général et même au stylisticien d'auteur. Ils vont apporter à la littérature et à l'explication de textes le moyen de découvrir le sommet expressif de l'énoncé et une méthode utilisable dans l'analyse de son architecture expressive. Ils serviront d'instrument à la recherche du niveau expressif propre à telle langue. Tous les passages établis par Vinay et Darbelnet - le chassé-croisé, l'étoffement des prépositions, les niveaux de langue, les équivalences sémantiques, les adaptations, les modulations, les transpositions, etc. - peuvent et doivent être examinés et échelonnés selon la hiérarchie expressive. L'on viendra, ce faisant, à se rendre compte qu'une analyse sémantique du texte français et anglais n'est plus suffisante. Il nous faut aussi l'équivalence expressive déterminée par les démarches des deux langues.

YVONNE ST-PIERRE FARINA 